\title{
Special Issue on Charles Hamblin
}

\section{Edited by Douglas WaLton \& RaLPh H. JohnSON}

\section{Editors' Introduction}

Charles Leonard Hamblin was professor of philosophy at the University of New South Wales in Sydney Australia between 1955 and his premature death in 1985. He is best known in philosophy for his book Fallacies, the best book written on this subject since the time of Aristotle.

The subject of informal fallacies has been a constant presence in logic textbooks from the time of Aristotle, and its continuing importance is recognized by its prominent inclusion in the hundreds of textbooks on informal logic written in the last half of the 20th century and continuing to be written up to this day. However, as Hamblin made clear in Fallacies, the subject matter was sadly neglected over the history of logic, so much so that it is fair to say that the advances that were made during the last half of the 20th century were made mainly in the textbooks themselves. Yet the textbooks struggled with the subject matter, and their treatment of the topic lacked consistency, theoretical organization, and the depth of treatment needed to make the methods they proposed very effective. It was clear that Hamblin's strategy was the one recognized by scientists (but not often enough put into practice and carried though) of picking as a topic for study an area that is problematic in its current state of development, understudied, and badly in need of investigation.

The results of Hamblin's carrying out this strategy are remarkable. Although the subject is still neglected as an area of study in philosophy graduate schools, and the teaching of the important courses of informal logic are too often left to junior faculty and graduate students, there has been a remarkable growth of research in what is now called the interdisciplinary field of argumentation. Fallacies are one area of research in this field, but Hamblin's work had another impact on it as well. Through his chapter on formal dialogue systems in Fallacies (1970) and his paper on mathematical models of dialogue (1971), Hamblin built formal foundations that provided an approach to rational thinking that was a radical departure from the notions of argument and justification then current in philosophy and still dominant today. This current view of rationality is the belief-desire-intention (BDI) model, which specifies that rationally justifying a claim that is subject to doubt is carried out by a rational agent (or group of them) that updates its beliefs, and uses these beliefs to justify a proposition that can then be accepted as knowledge.

Hamblin's vision was radically different from this view. He advocated a dialogue-based model of rational acceptance that has been

(C) Douglas Walton and Ralph H. Johnson. Informal Logic, Vol. 31, No. 4, (2011), pp. i-iv. 
called the commitment model. On this model, two participants interact with each other in a dialogue in which each takes turns making moves in the form of speech acts, such as making an assertion or putting forward an argument. Each party has a commitment set and as moves are made, propositions are inserted into or retracted from each set. A commitment is a proposition that an agent has gone on record as accepting, and hence the two terms 'commitment' and 'acceptance' are very close to being equivalent in meaning. However, as shown in a classic collection of essays - the proceedings of a conference on acceptance and belief (Engel, 2000)-attempts to draw a precise and widely agreed-upon distinction between commitment and belief have been unsuccessful. The source of the problem appears to be the failure to reach basic agreement on how to define the notion of belief.

Hamblin's theory was not altogether new or unprecedented, for it is based on the ancient Greek idea associated with democracy that an intelligent debate which examines the evidence on both sides of an issue is the best way to decide what to do when deliberating or when debating an issue on what to accept as representing the truth of the matter. Both Plato and Aristotle thought this method of argumentation by pro and con arguments to be fundamentally important not only in civic life, but as a serious philosophical method. However, this method has been sadly neglected. According to Toulmin's account, the cause was the rise of science during the Enlightenment, with the success of the experimental method in science leading to a positivist mindset (Toulmin, 1958). Only rarely did scientists, Galileo being the leading example, take the dialogue method seriously as a form of inquiry.

On Hamblin's model, which can be called a dialectical model in historical perspective, a disputed claim is shown to be acceptable if the arguments both for and against it are brought forward and allowed to interact with each other in a rule-governed dialogue in which it is shown that the pro side has the stronger argument. This view provides the foundational basis for much of the current research in the interdisciplinary field of argumentation studies. The most distinctive difference between the commitment model and the BDI model is that the BDI model has as its basic building blocks desires and beliefs that are private psychological notions internal to an agent, while the dialectical model is based on commitments that are statements externally and verbally accepted by an agent in a communicative context.

It is less well known to philosophers that Hamblin was also a contributor to the field of computing. During the period in the 1950s when he worked with a computer company in Australia he wrote a programming language based on reverse Polish notation and created a compiler that translated the programs into the machine language of the DEUCE computer. Hamblin's work on this project had an impact on the development of stack-based computers. As described in a short biography initially commissioned by the Australian Computer $\mathrm{Mu}$ seum Society, "His main early contributions to computing, which date from the mid 1950s, were the development and application of reverse Polish notation and the zero-address store. He was also the developer 
of one of the first computer languages, GEORGE." It is also noted that his ideas have become "influential in the design of computer interaction protocols, and are expected to shape the next generation of ecommerce and machine-communication systems."

Today the field of computing has embraced argumentation as a paradigm for research in artificial intelligence and multi-agent systems, spreading the impact of Hamblin's revolutionary views further. Throughout many of its areas, artificial intelligence has seen a prolific growth in uses of argumentation: agent system negotiation protocols, argumentation-based models of evidential reasoning and legal procedures, group work tools that use argument to structure debate, computer-based learning tools that use dialogical argument structures, argumentation models of deliberation for decision-making, and models of knowledge engineering using core concepts of argumentation (Besnard et al., 2008). Now the regular COMMA conference (the conference on Computational Model of Arguments) has become a regular forum for presenting research on the computational aspects of argumentation. According to the COMMA website, the conference "has provided a fruitful way of approaching non-monotonic and defeasible reasoning, deliberation about action, and agent communication scenarios such as negotiation." Among the application domains cited are law, medicine and computer-assisted democracy. Other major conferences in computer science now list argumentation as a main topic category for submitting papers, including even specialized areas like argumentation schemes, argument-based negotiation, decision-making based on argumentation dialogues, and argumentation systems for collaborative learning.

Although Hamblin's contributions to philosophy are well enough recognized, and his early contributions to computing have been acknowledged, it seems strange that he is not widely recognized for the powerful interdisciplinary effect his work on dialogue systems has had. There has been a back and forth process whereby his theories on formal dialogue systems and imperatives were taken up in argumentation, then used in computing, then transferred back to argumentation where computational tools, like those for argument mapping and visualization, have proved useful in philosophy, logic and a wide variety of other fields.

It is unfortunate that Hamblin's contributions in this regard still do not get him the credit he deserves for his remarkable achievements. In a recent announcement of a Special Issue of Journal of Logic and Computation entitled "20 years of argument-based inferences" (accessed October 07, 2011), we are told, "the field of formal argumentation can be traced back to work published in the early nineties." 2 This view of the origins of the field of formal argumentation has been widely circulated in the field of computing. It is, however, historically inaccurate. The field of formal argumentation traces directly back to Hamblin's work, especially the chapter on formal dialogue systems in his book on fallacies, his paper on mathematical dialogue structures,

\footnotetext{
${ }^{1}$ http://www.vukutu.com/blog/2011/01/a-salute-to-charles-hamblin/

2 http://jlcabi11.ing.unibs.it/
} 
and his book on imperatives, some 20 years earlier. The erroneous view needs to be corrected. The leading analytical philosophers of that period analyzed rational thinking using the model of a solitary agent updating its belief (BDI model). The work in the nineties had recognized defeasibility, but had not recognized the explicit idea of what has now emerged as the defining characteristic of argumentation, a dialogue in which two parties take turns making moves in putting forward speech acts. This was a radical idea at the time, something out of the mainstream that later turned out to be both a paradigm shift for philosophy and a conceptual breakthrough for computing.

We conclude this introduction with a brief bibliography of some of Hamblin's most prominent works. A comprehensive bibliography can be found in "A Salute to Charles Hamblin" (see footnote on p. iii).

\section{References}

Besnard, P, Doutre, S. and Hunter, A. (2008). Proceedings of the 2008 Conference on Computational Models of Argument. Amsterdam: IOS Press.

Engel P. (2000) (ed.). Believing and Accepting. Dordrecht: Kluwer.

Toulmin, S.E. (1958). The Uses of Argument. Cambridge: Cambridge University Press.

\section{Some Works of Charles Hamblin}

Hamblin, C.L (1959). The modal 'probably.' Mind 68: 234-240.

Hamblin, C.L. (1962). Translation to and from Polish notation. Computer Journal 5: 210-213.

Hamblin, C.L. (1963). Questions aren't statements. Philosophy of Science 30(1): 62-63.

Hamblin, C.L. (1966). Elementary Formal Logic, a Programmed Course. (Sydney: Hicks Smith). Republished, London: Methuen, 1967.

Hamblin, C.L. (1970). Fallacies, London: Methuen.

Hamblin, C.L. (1971). Mathematical models of dialogue. Theoria, 37: 130-155.

Hamblin, C.L. (1987). Imperatives. Oxford: Blackwell. 